\title{
Preparedness of pharmacists to respond to the emergency of the COVID-19 pandemic in Brazil: a comprehensive overview
}

\author{
Maria Auxiliadora Parreiras Martins $s^{1,2,3,4}$ (1) $\cdot$ Amanda Fonseca de Medeiros $^{2} \cdot$ Claudmeire Dias Carneiro de Almeida $^{3}$. \\ Adriano Max Moreira Reis ${ }^{1,2,3,4}$
}

Published online: 31 July 2020

(c) Springer Nature Switzerland AG 2020

\begin{abstract}
The outbreak of COVID-19 in low- and middle-income countries is worrisome due to the social inequalities in these countries, their limited health budgets and the significant burden of other acute and chronic diseases. The leap in the number of cases in Brazil has imposed a huge strain on the healthcare system. We sought to provide a comprehensive overview of the challenges encountered by pharmacy services in responding to the COVID-19 pandemic emergency in Brazil and discuss the role of clinical pharmacists in this context. Pharmaceutical services play a key role in the emergency response to the pandemic. The pharmacy workforce has been actively working to manage drug shortages, redesign workflow, and review drug formularies/protocols to improve safety for patients and healthcare professionals (HCPs). COVID-19 patients may present high risk in the use of medications and clinical pharmacists can contribute substantially as part of a multidisciplinary team to improve outcomes in drug therapy in severe and critical illness. The participation of pharmacists as members of antimicrobial stewardship programs should be enhanced to ensure appropriate and safe use of antibiotics in this context. HCPs should be encouraged to seek improvements in the performance of pharmaceutical services and innovative practices to respond to the pandemic. Further studies are needed to generate knowledge on COVID-19 to improve patient care in vulnerable populations.
\end{abstract}

\section{Introduction}

Coronavirus disease 2019 (COVID-19) is an emerging infectious disease first described in Wuhan, China, in December 2019, caused by the novel severe acute respiratory syndrome coronavirus (SARS-CoV-2) [1]. The outbreak of COVID-19 spread rapidly worldwide, being declared a pandemic on 11 Mar 2020 by the World Health Organization (WHO). Human-to-human transmission occurs via droplets or

Maria Auxiliadora Parreiras Martins

auxiliadorapmartins@hotmail.com

1 Faculdade de Farmácia, Universidade Federal de Minas Gerais, Av. Pres. Antônio Carlos, 6627, Campus Pampulha, Belo Horizonte, Minas Gerais 31270-901, Brazil

2 Hospital Risoleta Tolentino Neves, R. das Gabirobas, 1, Vila Cloris, Belo Horizonte, Minas Gerais 31744-012, Brazil

3 Hospital das Clínicas da Universidade Federal de Minas Gerais, Av. Prof. Alfredo Balena, 110, Bairro Santa Efigênia, Belo Horizonte, Minas Gerais 30130-100, Brazil

4 Instituto para Práticas Seguras no Uso de Medicamentos, ISMP-Brasil, Av. do Contorno, 9215, sl 502, Bairro Prado, Belo Horizonte, Minas Gerais 30110-063, Brazil contaminated hands or surfaces [1]. Various levels of classic public health strategies, such as social distancing, isolation, quarantine, and community containment, have been applied across countries to reduce the dissemination of the disease [2].

By July 2020, SARS-CoV-2 had infected $>14$ million people in 216 countries, creating an enormous burden on healthcare systems and the economy [3]. It reached the southern hemisphere later than the other continents and the progress of its transmission in the winter 'flu' season is unclear. The infection spread in low- and middle-income countries (LMICs) is worrisome due to their inability to respond rapidly to a pandemic situation in a scenario of social inequalities, limited health budgets, and the significant burden of other acute and chronic diseases [4, 5].

The clinical spectrum of COVID-19 is heterogeneous, encompassing asymptomatic disease, mild upper respiratory tract infection, and severe viral pneumonia that may progress to critical illness and death $[6,7]$. Common signs of the disease include fever, myalgia, cough, and dyspnea [8]. Around $20 \%$ of confirmed COVID-19 cases develop severe or critical illness [6], representing the subgroup of patients who are challenging frontline healthcare professionals (HCPs) 
in hospitals. Prognosis is poor for older adults [9] and in the presence of comorbidities, such as cardiovascular diseases $[8,10]$.

Knowledge regarding COVID-19 is being acquired at the bedside during the accelerated race to find efficacious drug treatments and vaccines. Worldwide, clinical trials have been designed to investigate the efficacy and safety of old and new drug candidates as treatments for COVID-19, but no guidelines have been established thus far [11]. In the context of assistance to COVID-19 patients, pharmacy services need to consider adjustments in drug supply, workflow, and pharmaceutical care. We sought to provide a comprehensive overview of the challenges encountered by pharmacy services to respond to the COVID-19 pandemic emergency and to describe its role in the Brazilian context.

\section{Unique features of the Brazilian response to COVID-19}

The first case of COVID-19 in Brazil was officially confirmed on 25 Feb 2020, and the infection has spread rapidly, infecting $2,012,151$ individuals by 18 July 2020 , with 76,688 deaths [3]. Brazil is in second place with regard to the number of cases and deaths, being declared by the WHO as the new epicenter of the coronavirus outbreak. Due to the low testing capacity, testing of severe cases has been prioritized and the total number of cases is still underestimated. According to the Brazilian Ministry of Health, the number of hospitalizations to manage acute respiratory distress syndrome (ARDS) was $>600 \%$ higher in 2020 than the same period in 2019 [12, 13]. The transmission of the virus is not yet controlled and Brazil has been reported to have the highest rate of transmission ( $R 0$ of 2.81) among 48 analyzed countries [14].

It is hypothesized that COVID-19 was imported by wealthy Brazilians who travelled abroad and then transmitted locally to poor communities, who mostly live in precarious conditions $[15,16]$. Social isolation and containment measures, as well as hygiene recommendations, are almost impossible to follow in these environments. Healthcare in Brazil is provided by public and private health systems. The first suspected and confirmed cases of COVID19 were referred to private services, but later poor people began to seek assistance in the public system. Brazil is a large South American country with 26 states with diverse social, cultural, and economic backgrounds. Hospital beds are unequally distributed across the country and most beds in intensive care units (ICUs) are concentrated in the Southeast region. People requiring ventilation support may outnumber the available ventilators and ICU beds [5]. In this context, COVID-19 remains unequally spread in a country with a political scenario of uncertainties [17], involving instability in the Ministry of Health and polarization in the political and ideological fields.

To provide assistance of quality in the middle of a crisis is extremely challenging, especially if we consider the particularities of our population. Prior to the emergence of COVID-19, Brazil had undergone a transition in epidemiological age, related to decreasing mortality and increasing life expectancy [18], with a projected increase in the population aged $>60$ years of almost threefold by 2050 [19]. The burden of chronic conditions, including diabetes mellitus, cardiovascular diseases (e.g., ischemic heart disease and stroke), cancer, and obesity is growing. Additionally, the public healthcare system still needs to cope with chronic infectious diseases, including HIV/AIDS, tuberculosis, syphilis, and malaria, and also endemic and emerging vector-borne arboviruses, such as yellow fever, dengue, Zika, and chikungunya [18].

In late March 2020, scheduled elective healthcare procedures and appointments started being gradually cancelled within Brazilian states to prevent virus transmission. Strategies have been implemented to reorganize the available beds to assist COVID-19 patients and other emergency clinical and surgical patients, and increase the number of beds by building temporary field hospitals where necessary. Healthcare settings have restructured workflow, adapting protocols to address new requirements of biosafety and providing training to staff. Despite the lack of conclusive scientific evidence, protocols are being rapidly updated to improve safety for patients and HCPs. Pharmaceutical services have been encouraged to be actively involved in various steps of these processes.

\section{Pharmaceutical services' preparedness and response}

The early experience of pharmaceutical services during the outbreak of COVID-19 in China highlighted the indispensable work of community [20,21] and hospital [22] pharmacists in the emergency response to the pandemic [23]. The International Pharmaceutical Federation (FIP) released the "Coronavirus 2019-nCoV Outbreak: Information and Interim Guidelines for Pharmacists and the Pharmacy Workforce", drafted with the participation of the Chinese Pharmaceutical Association (CPA), to provide reliable information on the management of pharmacy operations [24]. The Pharmacy Emergency Preparedness and Response (PEPR) recommendations were published to guide pharmacy endeavors towards full integration within public health and in recognition of pharmacists' skills, roles, and contributions as integral members of multidisciplinary healthcare teams [25]. These references can be used to create regional guidance 
for pharmacists and adapted to local demands and resources. The Brazilian Society of Hospital Pharmacy released recommendations for pharmacists working in different scenarios to fight against the pandemic [26].

An economic disruption without precedent has occurred together with a dramatic increase in the consumption of health products worldwide. Global drug shortages were triggered by inadequate quantities of raw materials, procurement and manufacturing issues, and drug discontinuations [27]. In Brazil, drug shortages also involved importation difficulties, exorbitant costs, compulsory advance payments, and noncompliance with agreed quantities and delivery deadlines. The supply of essential drugs has been jeopardized, including sedatives (fentanyl and midazolam) and neuromuscular agents (suxamethonium, atracurium). Commitment of pharmacists is required to manage drug shortages and minimize the impact on patient outcomes [23, 25, 27, 28].

The Brazilian population has been impacted by a flurry of fake news. The fear of catching COVID-19 and free access to drugstores have caused a rush to buy medicines for selftreatment. Data presented by the Federal Board of Pharmacy revealed a substantial increase in drug sales involving ascorbic acid (180.0\%), acetaminophen (77.4\%) and hydroxychloroquine sulfate (67.9\%) from January to March 2020 relative to the same period in 2019 [29]. The off-label use of chloroquine/hydroxychloroquine for preventing or treating COVID-19 imposes safety issues and requires the implementation of prescription monitoring strategies [30]. Panic buying of alcohol-based formulations, face shields, N95 respirators and other personal protective equipment (PPE) has also occurred, leading to shortages in hospitals and other healthcare facilities. The supply has been partially restored after government interventions and the recommendation to the public to wear fabric face masks. Population health interventions and the support of pharmacists for the rational use of drugs was shown to be of utter importance in this scenario. The Brazilian branch of the Institute for Safe Medication Practices (ISMP-Brasil; https://www.ismp-brasil.org/ site/) has provided important contributions for this approach.

Another concern is the effect of COVID-19 on increasing morbimortality of patients with chronic diseases, given their compromised access to healthcare and diagnostic tests. Delays in assessment may worsen the prognosis of chronic diseases. Kretchy et al. [31] discussed the challenges of managing chronic diseases and promoting medication adherence in LMICs. Community pharmacists were identified as front-line personnel to promote healthy lifestyle adjustments, refill prescriptions, provide counseling on the management of chronic diseases, and minimize unsafe self-medication habits with over-the-counter medicines. Pharmacists working through telehealth services could enhance outcomes in drug therapy and mitigate strategies to reduce the risks of COVID-19 contagion, such as triaging people with flu-like symptoms and improving workflow for physical distancing and self-protection [25, 32, 33]. Strategies to support the public and HCPs to cope with stress, anxiety, and poor sleep quality during the pandemic have been reported worldwide [25, 33, 34], and remote services have gradually started to operate with this aim in Brazil. However, the number of people with access to remote assistance is still limited.

Pharmaceutical services have been adjusting to respond to the COVID-19 outbreak at the level of public and private institutions, with attempts to meet the needs of patient care in each Brazilian region, according to the available financial and human resources. HCPs and students have been recruited by the Ministry of Health through the program "Brasil conta comigo" ('Brazil counts on me') as volunteers to face the pandemic. Basheti et al. [35] investigated the pharmacists' and pharmacy students' awareness of their role during this emergent situation in Jordan and the source of their information on COVID-19. In Brazil, the education and training of pharmacy professionals and students are under intense discussion in universities and healthcare settings. Live broadcasts, remote trainings and recorded courses using the internet and other media are being used as important means of education.

Pharmacists have been working on laboratory testing for COVID-19, administration of pharmacy supplies and replenishment, and also patient care, replicating experiences in other countries [20, 23, 33, 36]. Attributes of pharmaceutical services in the community and hospital settings in Brazil are summarized in Fig. 1. In community pharmacies, actions are focused on patients, comprising provision of information on COVID-19, promotion of the safe use of medicines, and guidance on medication adherence in acute and chronic diseases. In hospital pharmacies, beyond interactions with patients, pharmacists develop integrated actions with multidisciplinary teams in different fields of expertise, such as emergency and intensive care. Hospital pharmacists, in partnership with infection control committees, have produced remarkable results in the internal review of work processes, including rotation of working hours, home offices, distancing between workers, intensification of environmental control measures and self-protection of workers, new guidance on the use of sanitizers, and training on good practices for self-care at home, on the move, and at work. Hospital pharmacies seek to optimize clinical, humanistic, and economical outcomes in drug therapy by performing medical history and drug therapy reviews, patient follow-up, and medication reconciliation to ensure safe transition of care, as well as participating in antimicrobial stewardship programs (ASPs).

Pharmacists in community and hospital settings should enhance medication safety strategies and precautions in special populations with the aim of reducing avoidable harm related to medications. The development and refinement of pharmacists' role in the community and hospital settings is 


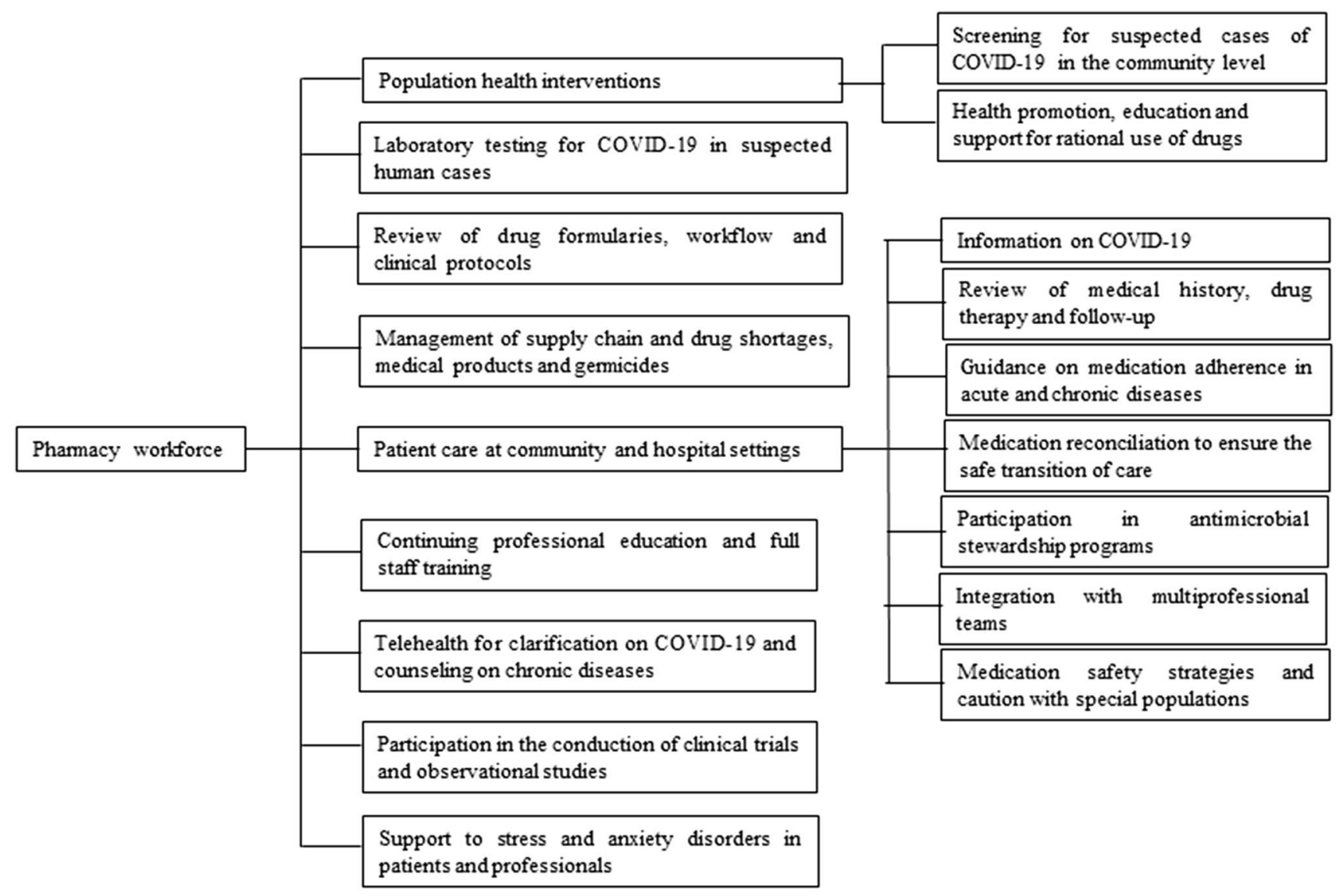

Fig. 1 Role of pharmaceutical services during the COVID-19 pandemic in Brazil

heterogeneous across regions. However, establishing robust organization of pharmacy services with clear planning of structure, processes, and outcomes' assessment [25] could contribute to ensuring the quality of the response to the pandemic. Reimbursement issues need to be addressed and advanced in Brazil.

\section{Clinical implications for pharmaceutical care}

The pharmacy workforce need to understand the nature of COVID-19, how it is transmitted, and how to prevent its spread [24]. Clinical pharmacists should constantly seek updated knowledge on COVID-19 to improve the monitoring of effectiveness and safety of drug therapy, and to promote productive interaction within the multidisciplinary team [25]. However, at the time of writing, high-quality evidence to support pharmacologic treatments for COVID-19 is still lacking [37]. Whilst waiting the results of ongoing clinical trials, experimental treatments are being used worldwide. Various protocols have been proposed by different committees and institutions in Brazil. On 18 May, a consensus of the Brazilian Association of Intensive Medicine, Brazilian
Society of Infectology and Brazilian Society of Pneumology and Tisiology was published as a reference for the pharmacological treatment of COVID-19 [38]. Off-label drugs currently used in Brazil include hydroxychloroquine (chloroquine) solely or in combination with azithromycin, lopinavir/ritonavir/oseltamivir, tocilizumab, glucocorticoids and parenteral anticoagulation. Due to conflicting scientific evidence, HCPs should reach a consensus, with decisions on drug therapy being individualized, based on disease stage, risk/benefit assessment, and pharmacoeconomic aspects.

Pharmacovigilance is needed to monitor the safety of experimental treatments and provide up-to-date guidance to HCPs. For instance, increasing numbers of patients with ARDS are receiving empirical treatment with oseltamivir due to limitations of COVID-19 testing, which may increase the risk of drug toxicity. In Brazil, the National Health Surveillance Agency (Agência Nacional de Vigilância Sanitária; ANVISA) is responsible for evaluating adverse drugs events (ADE) notifications made through the Vigimed $^{\circledR}$ system. Clinical pharmacists play a key role in the identification and reporting of these ADE, especially in the critically ill.

Individuals with severe or critical COVID-19 should be considered high-risk patients with regard to the use of 
potentially beneficial therapies and should be closely monitored by clinical pharmacists. Liver and kidney injuries in COVID-19 may alter the pharmacokinetics of drugs currently prescribed to treat the disease. Lopinavir/ritonavir/ oseltamivir, hydroxychloroquine and chloroquine are metabolized in the liver and most of their metabolites depend on renal excretion. Therefore, injury to the liver and kidneys can hinder metabolism, excretion, and the achievement of desirable serum concentrations, increasing the risk of drug toxicity [39]. Pre-existent liver and kidney diseases, impairment of these organs in older adults, and the presence of comorbidities can elevate the risk of clinical complications in COVID-19 [10, 39-41].

Other parameters to monitor effectiveness and safety of drug therapy should be included in clinical pharmacy protocols. Thromboprophylaxis in COVID-19 using non-fractionated heparin or low-molecular-weight heparins has been used to control the hypercoagulability state in hospitalized patients [42, 43], with multiple pharmacological mechanisms previously described $[44,45]$. Risk-benefit assessments of anticoagulation therapy should consider individual contraindications, stratification of bleeding risk, and dosage adjustments in patients with renal impairment and obesity [46]. The use of proarrhythmic medications should also be closely monitored. Patients receiving hydroxychloroquine or chloroquine \pm azithromycin should be frequently monitored for cardiac toxicity by checking their corrected QT (QTc) interval and for the use of other medications with a risk of QTc interval prolongation or torsade de pointes [47].

Additionally, the empiric use of antibiotics for suspected secondary bacterial infections needs to be optimized on initiation and rigorously de-escalated [48, 49]. Hospitals should not lose sight of the long-term threat of antimicrobial resistance, if their current structures and ASPs are disrupted during the pandemic [50]. Hence, the participation of clinical pharmacists with advanced infection disease training as members of ASPs is fundamental in ensuring appropriate and safe use of antibiotics and improvements in the quality of care. However, the involvement of ASPs in the COVID19 response is still incipient despite being recommended by experts in this field [51]. The use of medications should take into account special populations, including pregnant [52], pediatric [53], breastfeeding [54], and cancer patients [55]. The desirable attributes of clinical pharmacists to provide care for hospitalized COVID-19 patients in Brazil are summarized in Table 1.

Due to the lack of definitive protocols, the management of COVID-19 has been primarily supportive [49]. The FASTHUG-MAIDENS mnemonic (Table 1) is a standardized structured approach for pharmacists to identify drug-related problems in ICUs [56]. Several authors have described potential markers of critical illness, staging, or disease recovery in COVID-19 (e.g., D-dimer, lactate dehydrogenase, interleukin-6) [8, 10, 43, 57-60]. The course of an intense inflammatory process leads to alterations in many

Table 1 Attributes of pharmaceutical care provided to hospitalized COVID-19 in Brazil

\author{
Routine activities for clinical pharmacists \\ Review of patients' medical history \\ Provision of real-time assessment and evidence-based (when possible) advice on drug therapy \\ Support on safe use of medications brought from home \\ Medication reconciliation at different levels of transition of care \\ Simplification of drug administration schedule to reduce the exposure of nurses to COVID-19 patients \\ Monitoring of potential drug-drug, drug-food interactions and adverse drug reactions \\ Adjustments in dosing regimens according to liver and kidney functions \\ Prevention of medication errors \\ Optimization of drug therapy and electrolytes to minimize the risk of prolonged corrected QT intervals and torsade de pointes \\ Support on lung-protective ventilation and neuromuscular blocking agents to facilitate ventilator synchrony \\ Provision of conservative fluid strategies and monitoring of vasopressors use \\ Monitoring of empirical antibiotics for suspected bacterial co-infection with rigorous de-escalation \\ Employment of FASTHUG-MAIDENS mnemonic to identify drug-related problems in intensive care units \\ Support on drug information to patients and multidisciplinary teams, following biosafety protocols \\ Considerations on special situations (pediatrics, older adults, people with chronic diseases, allergies) \\ Research and continuing education \\ Precise documentation of pharmaceutical interventions
}

COVID-19 Coronavirus disease 2019, FASTHUG feeding, analgesia, sedation, thromboprophylaxis, hyperactive or hypoactive delirium, MAIDENS medication reconciliation, antibiotics or anti-infectives, indications for medications, drug dosing, electrolytes, hematology and other laboratory results, no drug interactions, allergies, duplications, side effects, stop dates 
Table 2 Panel of laboratory tests with potential utility for monitor ing patients with COVID-19

Laboratory tests (units; may vary according to the method adopted)

Blood biochemistry

Total protein $(\mathrm{g} / \mathrm{L})$

Albumin $(\mathrm{g} / \mathrm{L})$

Total bilirubin $(\mu \mathrm{mol} / \mathrm{L})$

$\gamma$-Glutamyltranspeptidase (U/L)

Alkaline phosphatase (U/L)

Lactate dehydrogenase (U/L)

Blood urea nitrogen $(\mathrm{mmol} / \mathrm{L})$

Aspartate aminotransferase $(\mathrm{U} / \mathrm{L})$

Alanine aminotransferase (U/L)

Serum creatinine $(\mu \mathrm{mol} / \mathrm{L})$

Total creatine kinase $(\mathrm{U} / \mathrm{L})$

Interleukin-6 (pg/mL)

Infection biomarkers

Procalcitonin $(\mathrm{ng} / \mathrm{mL})$

C-reactive protein $(\mathrm{mg} / \mathrm{L})$

Serum ferritin $(\mathrm{ng} / \mathrm{mL})$

Blood routine

Red blood cell count (per $\mathrm{mm}^{3}$ )

Hemoglobin (g/dL)

Hematocrit (\%)

Leukocytes count (per $\mathrm{mm}^{3}$ )

Neutrophils $\left(1 \times 10^{9} / \mathrm{L}\right)$

Lymphocytes $\left(1 \times 10^{9} / \mathrm{L}\right)$

Neutrophil-lymphocyte ratio

Platelet count (per $\mathrm{mm}^{3}$ )

Electrolytes

Sodium (mmol/L)

Potassium (mmol/L)

Magnesium (mmol/L)

Chlorine $(\mathrm{mmol} / \mathrm{L})$

Calcium (mmol/L)

Coagulation function

Prothrombin time (s)

Activated partial thromboplastin time (s)

INR

Fibrinogen $(\mathrm{g} / \mathrm{L})$

Antithrombin activity (\%)

D-dimer $(\mu \mathrm{g} / \mathrm{mL})$

Fibrin degradation product $(\mu \mathrm{g} / \mathrm{mL})$

Cardiac biomarkers

BNP

Pro-BNP

Hypersensitive troponin I ( $\mathrm{pg} / \mathrm{mL})$

Arterial blood gas

$\mathrm{PaO}_{2} \pm$ oxygen inhalation $(\mathrm{mm} \mathrm{Hg})$

Serum bicarbonate $\left(\mathrm{HCO}_{3}\right)$

BNP B-type natriuretic peptide, COVID-19 coronavirus disease 2019, INR International Normalized Ratio, $\mathrm{PaO}_{2}$ partial pressure of oxygen laboratory tests in patients with acute or severe/critical illness. Specific guidance on the use of laboratory tests to predict critical illness in COVID-19 is under investigation [61]. Thus, to date, laboratory tests need critical interpretation and HCPs should make consensual decisions based on patients' clinical condition. Laboratory tests with potential utility to monitor COVID-19 in hospitals are listed in Table 2. In Brazil, the use of these tests is heterogeneous in healthcare settings, and may be limited due to cost issues, delayed release of results, and the lack of equipment or partnerships between institutions.

There are many unanswered questions regarding COVID19. Thrombotic risk varies according to race and ethnicity [62]; however, the impact of the heterogeneous ancestral contributions from African, Caucasian, and indigenous genomes in the Brazilian populations [5] are not yet clear. Regarding the use of medications, the protective effect of chronic use of oral anticoagulants and the clinical impact of ACE inhibitors and ARBs commonly prescribed to treat hypertension on the risks of COVID-19 are currently unclear $[40,49]$. Furthermore, the association between the complications of severe COVID-19 and Chagas disease and rheumatic heart diseases, conditions that are still prevalent in Brazil [63], is not established. We also do not know if the determinants of severity in Brazil will be similar to those observed in the USA [61], China, and Europe [58, 60], given other factors such as social inequality and endemic diseases. Future studies in Brazil should investigate clinical presentation, according to the features of inflammatory response, treatment outcomes, and the factors associated with critical illness.

\section{Take-home messages}

- Pharmacists play a key role in the management of COVID-19 in Brazil by providing health interventions and specialized support to improve outcomes, especially in populations with underlying diseases and social inequalities.

- Pharmaceutical expertise has been relevant in managing drug shortages, redesigning workflow, and educating pharmacists in Brazil.

- Pharmacists can contribute to multidisciplinary teams, including antimicrobial stewardship programs, to improve outcomes of drug therapy in severe and critically ill patients.

- Further studies are needed to improve COVID-19 knowledge and patient care in low- and middle-income countries. 
Acknowledgements This study was partially supported by the Universidade Federal de Minas Gerais, Hospital Risoleta Tolentino Neves, and Hospital das Clínicas of the Universidade Federal de Minas Gerais.

Author contributions MAPM, AFM, CDCA, and AMMR made substantial contributions to the conception and design of the work. MAPM drafted the manuscript. All authors participated in the interpretation of data. They provided a critical review of the manuscript and approved its final version. All authors agree to be accountable for all aspects of the work and in ensuring the integrity of any part of the work.

\section{Compliance with ethical standards}

\section{Funding None.}

Conflict of interest The authors declare that they have no conflict of interest.

\section{References}

1. World Health Organization. Novel coronavirus - China. Geneva: World Health Organization; 2020.

2. Wilder-Smith A, Freedman DO. Isolation, quarantine, social distancing and community containment: pivotal role for old-style public health measures in the novel coronavirus $(2019-\mathrm{nCoV})$ outbreak. J Travel Med. 2020;27(2):taaa020.

3. World Health Organization. Coronavirus disease (COVID-19). Situation report - 180. https://www.who.int/emergencies/diseases/ novel-coronavirus-2019/situation-reports/. Accessed 19 July 2020.

4. Hopman J, Allegranzi B, Mehtar S. Managing COVID-19 in lowand middle-income countries. JAMA. 2020;323(16):1549-50.

5. Marson FAL, Ortega MM. COVID-19 in Brazil. Pulmonology. 2020;S2531-0437(20):30087-8.

6. Chinese Center for Disease Control and Prevention (CCDC). The epidemiological characteristics of an outbreak of 2019 novel coronavirus diseases (COVID-19). China. 2020;2(8):113-22.

7. Bai Y, Yao L, Wei T, et al. Presumed asymptomatic carrier transmission of COVID-19. JAMA. 2020;323(14):1406-7.

8. Zhou F, Yu T, Du R, et al. Clinical course and risk factors for mortality of adult inpatients with COVID-19 in Wuhan, China: a retrospective cohort study. Lancet. 2020;395(10229):1054-62.

9. Verity R, Okell LC, Dorigatti I, et al. Estimates of the severity of coronavirus disease 2019: a model-based analysis. Lancet Infect Dis. 2020;20(6):669-77.

10. Yang X, Yu Y, Xu J, et al. Clinical course and outcomes of critically ill patients with SARS-CoV-2 pneumonia in Wuhan, China: a single-centered, retrospective, observational study. Lancet Respir Med. 2020;8(5):475-81.

11. Kupferschmidt K, Cohen J. Race to find COVID-19 treatments accelerates. Science. 2020;367(6485):1412-3.

12. Ministry of Health of Brazil, Health Surveillance Department [Ministério da Saúde do Brasil, Secretaria de Vigilância Sanitária]. Special epidemiologic report COE-COVID-19 [in Portuguese]. https://portalarquivos.saude.gov.br/images/pdf/2020/ May/09/2020-05-06-BEE15-Boletim-do-COE.pdf. Accessed 7 May 2020.

13. Cimerman $\mathrm{S}$, Chebabo A, Cunha CAD, et al. Deep impact of COVID-19 in the healthcare of Latin America: the case of Brazil. Braz J Infect Dis. 2020;24(2):93-5.

14. Latin American Business Stories. Coronavirus contagion rate in Brazil is 2.8 , the highest among 48 countries, points out Imperial
College. https://labs.ebanx.com/en/notes/imperial-college-brazi 1-covid/. Accessed 7 May 2020.

15. Candido DDS, Watts A, Abade L, et al. Routes for COVID-19 importation in Brazil. J Travel Med. 2020;27(3):1-3.

16. The conversation. COVID-19 is deadlier for black Brazilians, a legacy of structural racism that dates back to slavery. https:// theconversation.com/covid-19-is-deadlier-for-black-brazilians -a-legacy-of-structural-racism-that-dates-back-to-slavery-13943 0 . Accessed 7 May 2020.

17. COVID-19 in Brazil: "So what?". Lancet. 2020; 395(10235): 1461.

18. GBD 2016 Brazil Collaborators. Burden of disease in Brazil, 1990-2016: a systematic subnational analysis for the Global Burden of Disease Study 2016. Lancet. 2018;392(10149):760-75.

19. United Nations Department of Economic and Social Affairs. World population ageing: 2017 highlights. New York: United Nations; 2017.

20. Zheng SQ, Yang L, Zhou PX, et al. Recommendations and guidance for providing pharmaceutical care services during COVID19 pandemic: a China perspective. Res Social Adm Pharm. 2020;S1551-7411(20):30284-9.

21. Ung COL. Community pharmacist in public health emergencies: quick to action against the coronavirus 2019-nCoV outbreak. Res Soc Adm Pharm. 2020;16(4):583-6.

22. Song Z, Hu Y, Zheng S, et al. Hospital pharmacists' pharmaceutical care for hospitalized patients with COVID-19: recommendations and guidance from clinical experience. Res Soc Adm Pharm. 2020. https://doi.org/10.1016/j.sapharm.2020.03.027.

23. Liu S, Luo P, Tang M, et al. Providing pharmacy services during the coronavirus pandemic. Int J Clin Pharm. 2020;42(2):299-304.

24. International Pharmaceutical Federation (FIP). Coronavirus SARS-CoV-2/COVID-19 pandemic: Information and interim guidelines for pharmacists and the pharmacy workforce (Updated 03-19-2020). https://www.fip.org/files/content/priority-areas/ coronavirus/COVID-19-Guidelines-for-pharmacists-and-thepharmacy-workforce.pdf. Accessed 7 May 2020.

25. Aruru M, Truong HA, Clark S. Pharmacy Emergency Preparedness and Response (PEPR): a proposed framework for expanding pharmacy professionals' roles and contributions to emergency preparedness and response during the COVID-19 pandemic and beyond. Res Soc Adm Pharm. 2020;S1551-7411(20):30323-5.

26. Brazilian Society of Hospital Pharmacy [Sociedade Brasileira de Farmácia Hospitalar - SBRAFH]. Contingency plan in various pharmaceutical scenarios of the COVID-19 pandemic [in Portuguese]. http://www.sbrafh.org.br/inicial/plano-de-contingenc ia-em-diversos-cenarios-farmaceuticos-no-ambito-da-pandemiapor-covid-19/. Accessed 7 May 2020.

27. Badreldin HA, Atallah B. Global drug shortages due to COVID19: impact on patient care and mitigation strategies. Res Social Adm Pharm. 2020;S1551-7411(20):30569-76.

28. Rosa MB, Reis AM, Perini E. Drug shortage: a public health problem. Cad Saude Publica. 2016;32(10):e00086916.

29. Federal Board of Pharmacy - Brazil [Conselho Federal de Farmácia-Brasil] survey shows how Covid-19's fear impacted medicine sales [in Portuguese]. http://www.cff. org.br/noticia.php?id $=5747 \&$ titulo $=$ Levantamento + mostr $\mathrm{a}+$ como+o+medo+da+Covid-19+impactou+venda+de+medic amentos. Accessed 7 May 2020.

30. Abena PM, Decloedt EH, Bottieau E, et al. Chloroquine and hydroxychloroquine for the prevention or treatment of novel coronavirus disease (COVID-19) in Africa: caution for inappropriate off-label use in healthcare settings. Am J Trop Med Hyg. 2020;102(6):1184-8.

31. Kretchy IA, Asiedu-Danso M, Kretchy JP. Medication management and adherence during the COVID-19 pandemic: perspectives 
and experiences from low-and middle-income countries. Res Soc Adm Pharm. 2020;S1551-7411(20):30332-6.

32. Yemm KE, Arnall JR, Cowgill NA. Necessity of pharmacistdriven non-prescription telehealth consult services in the era of COVID-19. Am J Health Syst Pharm. 2020. https://doi. org/10.1093/ajhp/zxaa162.

33. Li H, Zheng S, Liu F, et al. Fighting against COVID-19: innovative strategies for clinical pharmacists. Res Soc Adm Pharm. 2020. https://doi.org/10.1016/j.sapharm.2020.04.003.

34. Huang Q, Deng X, Li Y, et al. Clinical characteristics and drug therapies in patients with the common-type coronavirus disease 2019 in Hunan, China. Int J Clin Pharm. 2020;42(3):837-45.

35. Basheti IA, Nassar R, Barakat M, et al. Pharmacists' readiness to deal with the coronavirus pandemic: assessing awareness and perception of roles. Res Soc Adm Pharm. 2020;:S1551-7411(20):30418-26.

36. Meng L, Qiu F, Sun S. Providing pharmacy services at cabin hospitals at the coronavirus epicenter in China. Int J Clin Pharm. 2020;42(2):305-8.

37. Sanders JM, Monogue ML, Jodlowski TZ, et al. Pharmacologic treatments for coronavirus disease 2019 (COVID-19): a review. JAMA. 2020. https://doi.org/10.1001/jama.2020.6019.

38. Falavigna M, Colpari V, Stein C, et al. Guidelines for the pharmacological treatment of COVID-19. The task force/consensus guideline of the Brazilian Association of Intensive Care, the Brazilian Society of Infectious Diseases and the Brazilian Society of Pulmonology and Tisiology. Rev Bras Ter Intensiva. 2020;32(2):166-96.

39. Rismanbaf A, Zarei S. Liver and kidney injuries in COVID-19 and their effects on drug therapy; a letter to editor. Arch Acad Emerg Med. 2020;8(1):e17.

40. Shahid Z, Kalayanamitra R, McClafferty B, et al. COVID-19 and older adults: what we know. J Am Geriatr Soc. 2020;68(5):926-9.

41. Zhang Y, Geng X, Tan Y, et al. New understanding of the damage of SARS-CoV-2 infection outside the respiratory system. Biomed Pharmacother. 2020;127:110195.

42. Tang N, Bai H, Chen X, et al. Anticoagulant treatment is associated with decreased mortality in severe coronavirus disease 2019 patients with coagulopathy. J Thromb Haemost. 2020;18(5):1094-9.

43. Thachil J, Cushman M, Srivastava A. A proposal for staging COVID-19 coagulopathy. Res Pract Thromb Haemost. 2020. https ://doi.org/10.1002/rth2.12372.

44. Spiess BD. Heparin: effects upon the glycocalyx and endothelial cells. J Extra Corpor Technol. 2017;49(3):192-7.

45. Thachil J. The versatile heparin in COVID-19. J Thromb Haemost. 2020;18(5):1020-2.

46. Lee YR, Palmere PJ, Burton CE, et al. Stratifying therapeutic enoxaparin dose in morbidly obese patients by BMI class: a retrospective cohort study. Clin Drug Investig. 2020;40(1):33-40.

47. Sapp JL, Alqarawi W, MacIntyre CJ, et al. Guidance on minimizing risk of drug-induced ventricular arrhythmia during treatment of COVID-19: a statement from the Canadian Heart Rhythm Society. Can J Cardiol. 2020;36(6):948-51.

48. Campion M, Scully G. Antibiotic use in the intensive care unit: optimization and de-escalation. J Intensive Care Med. 2018;33(12):647-55.

49. Stringer KA, Puskarich MA, Kenes MT, et al. COVID-19: the uninvited guest in the intensive care unit (ICU) implications for pharmacotherapy. Pharmacotherapy. 2020;40(5):382-6.

50. Rawson TM, Moore LSP, Castro-Sanchez E, et al. COVID-19 and the potential long-term impact on antimicrobial resistance. J Antimicrob Chemother. 2020;75(7):1681-4.

51. Stevens MP, Patel PK, Nori P. Involving antimicrobial stewardship programs in COVID-19 response efforts: all hands on deck. Infect Control Hosp Epidemiol. 2020;41(6):744-5.

52. Rasmussen SA, Smulian JC, Lednicky JA, et al. Coronavirus disease 2019 (COVID-19) and pregnancy: what obstetricians need to know. Am J Obstet Gynecol. 2020;222(5):415-26.

53. Riphagen S, Gomez X, Gonzalez-Martinez C, et al. Hyperinflammatory shock in children during COVID-19 pandemic. Lancet. 2020;395(10237):1607-8.

54. Anderson PO. Breastfeeding and respiratory antivirals: coronavirus and influenza. Breastfeed Med. 2020;15(3):128.

55. Gosain R, Abdou Y, Singh A, et al. COVID-19 and cancer: a comprehensive review. Curr Oncol Rep. 2020;22(5):53.

56. Mabasa VH, Malyuk DL, Weatherby EM, et al. A standardized, structured approach to identifying drug-related problems in the intensive care unit: FASTHUG-MAIDENS. Can J Hosp Pharm. 2011;64(5):366-9.

57. Tang N, Li D, Wang X, et al. Abnormal coagulation parameters are associated with poor prognosis in patients with novel coronavirus pneumonia. J Thromb Haemost. 2020;18(4):844-7.

58. Wynants L, Van Calster B, Collins GS, et al. Prediction models for diagnosis and prognosis of COVID-19 infection: systematic review and critical appraisal. BMJ. 2020;369:m1328. https://doi. org/10.1136/bmj.m1328.

59. Wang F, Hou H, Luo Y, et al. The laboratory tests and host immunity of COVID-19 patients with different severity of illness. JCI Insight. 2020;5(10):137799.

60. Liang W, Liang H, Ou L, et al. Development and validation of a clinical risk score to predict the occurrence of critical illness in hospitalized patients with COVID-19. JAMA Intern Med. 2020. https://doi.org/10.1001/jamainternmed.2020.2033.

61. Petrilli CM, Jones SA, Yang J, et al. Factors associated with hospital admission and critical illness among 5279 people with coronavirus disease 2019 in New York City: prospective cohort study. BMJ. 2020;369:m1966. https://doi.org/10.1136/bmj.m1966.

62. Fogarty H, Townsend L, Cheallaigh CN, et al. COVID19 coagulopathy in Caucasian patients. $\mathrm{Br} \mathrm{J}$ Haematol. 2020;189(6):1044-9.

63. Marcolino MS, Palhares DM, Benjamin EJ, et al. Atrial fibrillation: prevalence in a large database of primary care patients in Brazil. Europace. 2015;17(12):1787-90. 